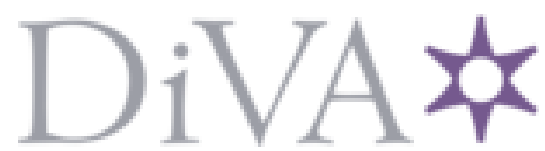

http://www.diva-portal.org

\title{
Postprint
}

This is the accepted version of a paper presented at 55th IEEE Conference on Decision and Control.

Citation for the original published paper:

Terelius, H., Johansson, K H. (2016)

On the optimal location of distribution centers for a one-dimensional transportation system.

In: Decision and Control (CDC), 2016 IEEE 55th Conference on IEEE

https://doi.org/10.1109/CDC.2016.7798650

N.B. When citing this work, cite the original published paper.

(c) 2016 IEEE. Personal use of this material is permitted. Permission from IEEE must be obtained for all other users, including reprinting/ republishing this material for advertising or promotional purposes, creating new collective works for resale or redistribution to servers or lists, or reuse of any copyrighted components of this work in other works.

Permanent link to this version:

http://urn.kb.se/resolve?urn=urn:nbn:se:kth:diva-194343 


\section{On the optimal location of distribution centers for a one-dimensional transportation system}

\author{
Håkan Terelius
}

\author{
Karl Henrik Johansson
}

\begin{abstract}
Transportation service providers are pressurized to enable real-time logistics planning from a constantly changing demand. This paper focus on a real-time transportation service provider operating along a one-dimensional highway. Transportation assignments arrive following a Poisson process, and the transportation service provider is operating on this road system with a fleet of vehicles, trying to minimize the expected delivery time. Specifically, the optimal locations for idle vehicles, and the optimal locations for construction of distribution centers are considered. The strategies are evaluated with numerical simulations along a Swedish highway system.
\end{abstract}

\section{INTRODUCTION}

The transportation system is vital for the economical development and functioning of our society, but it is facing great challenges adapting to increasing demands and requirements. In the EU the road transportation system consists of about 2 million trucks, and produces $18 \%$ of the total greenhouse gases [1], thus there are strong social, economical and sustainability motivations for making the transportation industry more efficient.

Real-time data gathering has been proved to increase the efficiency and flexibility in the planning of transport assignments, and this has spurred an active field of research into building automated highway systems [2, 3] over the last twenty years. The transition towards Justin-Time supply chains, employed to minimize the waste in the merchandise industry, is affecting the entire logistics chain [4] and transforming the requirements for the transportation industry. A consequence is the need for real-time transportation planning and adaption of transportation assignments [5, 6]. Large scale transportation service providers are also interested in optimizing the entire fleet management system [7], which also involves the design of distribution center locations [8, 9]. Related topics include the strategies for cruising taxis and dispatch systems [10, 11], repositioning in bike-sharing systems [12], and combinatorial pickup and delivery problems [13].

This paper focus on a real-time transportation service provider operating along a one-dimensional highway. The

The authors are with the ACCESS Linnaeus Centre, School of Electrical Engineering, KTH Royal Institute of Technology, SE-100 44 Stockholm, Sweden. Emails: \{ hakante, kallej \} @kth.se.

The research leading to these results has received funding from the European Union Seventh Framework Programme, under the Companion project. The research was also supported by the Swedish Research Council and the Knut and Alice Wallenberg Foundation. goal is to minimize the time it takes from the reception of a transportation assignment until delivery, and we especially consider the performance pertaining to the idling vehicles and the distribution center locations.

The outline of the paper is as follows. In section II, we introduce the one-dimensional road transportation model. In section III, we consider the optimal waiting locations for idling vehicles, and derive an explicit solution for uniformly random assignments. In section IV, we continue with the optimal locations for building distribution centers in order to minimize the traveling time, and similarly derive an explicit solution for uniformly random assignments, as well as an efficient algorithm for computing the locations for discrete random distributions. In section $\mathrm{V}$, we evaluate the strategies with numerical simulations, before concluding the paper in section VI.

\section{Transportation Model}

Consider a road freight transportation system between two cities, for example the main highway connecting the largest cities in Sweden as depicted fig. 1. A position along this route can be represented with its relative position in the interval $[0,1]$. Also, as shown in fig. 1 there are several major cities located along this road. Thus, using this model, any position of a vehicle, or destination is given by a number in the interval $[0,1]$. Furthermore, positions on the interval $[0,1]$ can be scaled such that the difference $\left|x_{1}-x_{2}\right|$ between two positions $x_{1}$ and $x_{2}$ is proportional to the transportation cost between these locations on the map, in terms of either travel distance, travel time or fuel consumption.

A real-time transportation provider is operating on this road system with a fleet of vehicles. Transportation assignments arrive randomly following a Poisson process with rate $\lambda$, and the pickup location $l_{1}$ and drop-off location $l_{2}$ are sampled from a joint probability density function $\rho\left(l_{1}, l_{2}\right):[0,1] \times[0,1] \rightarrow \mathbb{R}_{+}$, where we assume the transportation providers have prior knowledge about $\rho$. Each of the transportation provider's vehicles cycles through the states in fig. 2, where it starts in an idle state waiting for an assignment. After being selected for an assignment, it drives to the pickup location to collect the goods. The assignment is then brought to a distribution center, before being delivered to the drop-off location, after which the vehicle is returned to an idle state. 


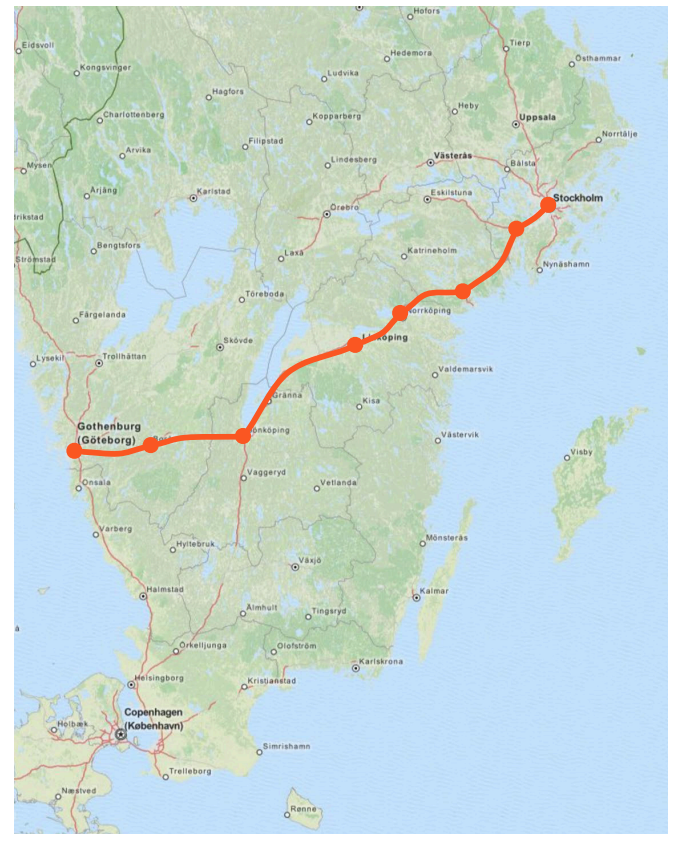

Figure 1. Map of southern Sweden, highlighting the main road connecting the largest cities Stockholm and Göteborg, together with the major cities along the road. (Map courtesy of OpenStreetMap)

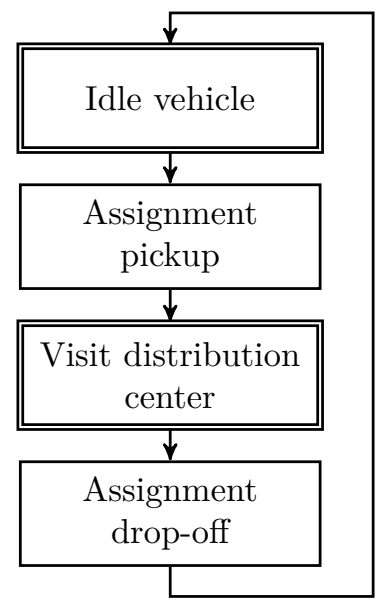

Figure 2. Flow chart of the states for each vehicle of a transportation provider. In this paper, we focus on optimizing the idle vehicle locations and the distribution center locations.

The transportation provider evaluates its performance as the time it takes from receiving a transportation assignment until the delivery at the drop-off location. This time can be divided into three parts, the time it takes for a vehicle to arrive at the pickup location, the time it takes to drive to the drop-off location, and the extra time spent visiting the distribution center. Here, the time taken to drive from the pickup location to the drop-off location is given by the assignment and road conditions, and is outside the control of the transportation provider, but the time to pickup an assignment depends on the location strategy for the idle vehicles, and the extra time spent going to a distribution center depends on where the distribution centers are built. In this paper, we consider both of these optimization problems in the next sections.

\section{Optimal IdLing Location}

In this section, the static optimization problem of deciding where idle vehicles should wait for their next assignment is considered. A transportation provider serving the system with $N$ vehicles would like to distribute the vehicles to minimize the expected time to pickup its next assignment, where the pickup location $l_{1}$ is randomly chosen from the probability density function $\rho\left(l_{1}\right):[0,1] \rightarrow \mathbb{R}_{+}$. Let $x_{1}, \ldots, x_{N}$ denote the locations of the transportation provider's $N$ vehicles, and $\mathbb{E}[\cdot]$ the expected value. The problem can then be formulated as

$$
\begin{aligned}
\min _{x_{1}, \ldots, x_{N}} \mathbb{E}_{l_{1}} & {\left[\min _{i=1, \ldots, N}\left|x_{i}-l_{1}\right|\right] } \\
& =\min _{x_{1}, \ldots, x_{N}} \int_{0}^{1}\left[\rho\left(l_{1}\right) \min _{i=1, \ldots, N}\left|x_{i}-l_{1}\right|\right] \mathrm{d} l_{1}
\end{aligned}
$$

Remark 1. In this formulation, the vehicles may stop at any location along the road, i.e., $x_{i} \in[0,1]$.

\section{A. Uniform distributions}

We will now derive an explicit solution for the locations of the vehicles, when the transport assignments have a uniform probability distribution.

Proposition 1. Assume that new transportation assignments arrive at locations following a uniform distribution $\mathcal{U}[0,1]$ over the road system, i.e., $\rho\left(l_{1}\right)=1$ for all $l_{1} \in[0,1]$. The optimal locations of the $N$ vehicles is then equidistantly distributed over the line, with $x_{i}=\frac{2 i-1}{2 N}$, $i=1, \ldots, N$.

Proof. Without loss of generality, assume that $x_{1} \leq x_{2} \leq$ $\cdots \leq x_{N}$. Thus, the integral can be split into parts as

$$
\begin{gathered}
\int_{0}^{1}\left[\min _{i=1, \ldots, N}\left|x_{i}-l_{1}\right|\right] \mathrm{d} l_{1} \\
=\int_{0}^{x_{1}}\left(x_{1}-l_{1}\right) \mathrm{d} l_{1}+ \\
\sum_{i=1}^{N-1}\left(\int_{x_{i}}^{\left(x_{i}+x_{i+1}\right) / 2}\left(l_{1}-x_{i}\right) \mathrm{d} l_{1}+\int_{\left(x_{i}+x_{i+1}\right) / 2}^{x_{i+1}}\left(x_{i}-l_{1}\right) \mathrm{d} l_{1}\right) \\
\quad+\int_{x_{N}}^{1}\left(l_{1}-x_{N}\right) \mathrm{d} l_{1} \\
=\underbrace{\frac{1}{2} x_{1}^{2}+\sum_{i=1}^{N-1} \frac{1}{4}\left(x_{i+1}-x_{i}\right)^{2}+\frac{1}{2}\left(1-x_{N}\right)^{2}}_{G}
\end{gathered}
$$

Thus, the vehicle locations $x_{1}, \ldots, x_{N}$ should be chosen such that $G$ is minimized, which happens when the gradient is zero. Solving this equation system yields the solution where $x_{i}=\frac{2 i-1}{2 N}$ for all $i=1, \ldots, N$. 


\section{Optimal Distribution Center Location}

In this section, the static optimization problem of deciding where to build distribution centers is considered. Distribution centers are used to store and sort goods, and to coordinate transportation assignments efficiently. We assume that every piece of goods need to visit a distribution center before being delivered to its final destination.

A transportation assignment consists of a pickup location $l_{1} \in[0,1]$ and a drop-off location $l_{2} \in[0,1]$, and the goods is transported from the pickup location to any distribution center before being delivered to the drop-off location. The goal is to decide where to build $M$ distribution centers such that the expected total transportation cost is minimized. Let $\rho\left(l_{1}, l_{2}\right):[0,1] \times[0,1] \rightarrow \mathbb{R}_{+}$be the joint probability density function for an assignment to have the pickup location $l_{1}$ and drop-off location $l_{2}$, and let $d_{1}, \cdots d_{M}$ be the locations for the distribution centers. The optimization problem can be formulated as

$$
\begin{gathered}
\min _{d_{1}, \ldots, d_{M}} \mathbb{E}_{l_{1}, l_{2}}\left[\min _{i=1, \ldots, M}\left(\left|d_{i}-l_{1}\right|+\left|d_{i}-l_{2}\right|\right)\right]= \\
\min _{d_{1}, \ldots, d_{M}} \int_{0}^{1} \int_{0}^{1}\left[\rho\left(l_{1}, l_{2}\right) \min _{i=1, \ldots, M}\left(\left|d_{i}-l_{1}\right|+\left|d_{i}-l_{2}\right|\right)\right] \mathrm{d} l_{2} \mathrm{~d} l_{1}
\end{gathered}
$$

\section{A. Uniform distributions}

Assume that the pickup and drop-off locations are i.i.d. random variables with uniform probability distribution $l_{1}, l_{2} \sim \mathcal{U}[0,1]$, i.e., $\rho\left(l_{1}, l_{2}\right)=1$. Let us first consider the case with only one distribution center.

Proposition 2. The optimal location, $d$, for a single distribution center, when the assignment locations have uniform probability density $\rho\left(l_{1}, l_{2}\right)=1$, is at $d=\frac{1}{2}$.

This is intuitively clear from a symmetry argument, but we will none the less prove it here.

Proof. The distribution center location $d$ is determined by the following optimization problem.

$$
\begin{aligned}
\min _{d} & \mathbb{E}_{l_{1}, l_{2}}\left[\left|d-l_{1}\right|+\left|d-l_{2}\right|\right] \\
& =\min _{d} \int_{0}^{1} \int_{0}^{1}\left(\left|d-l_{1}\right|+\left|d-l_{2}\right|\right) \mathrm{d} l_{2} \mathrm{~d} l_{1} \\
& =2 \min _{d} \int_{0}^{1}|d-l| \mathrm{d} l \\
& =2 \min _{d}\left(\int_{0}^{d}(d-l) \mathrm{d} l+\int_{d}^{1}(l-d) \mathrm{d} l\right) \\
& =2 \min _{d}\left(\frac{d^{2}}{2}+\frac{(d-1)^{2}}{2}\right) \\
& =\min _{d}\left(2 d^{2}-2 d+1\right)
\end{aligned}
$$

which has the solution $d=\frac{1}{2}$.

We now proceed to the general case, with $M>1$ distribution centers.

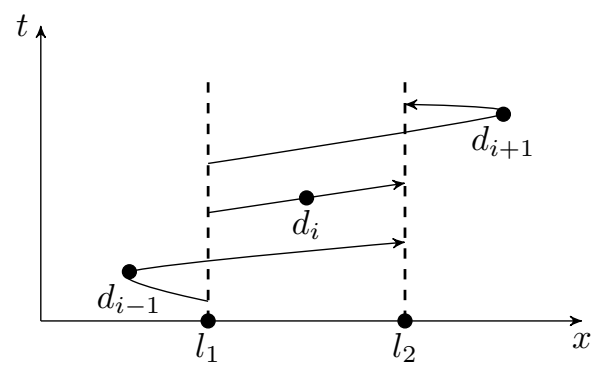

Figure 3. A schematic representation for the trip between $l_{1}$ and $l_{2}$, using three different possible distribution centers $d_{i-1}, d_{i}$ or $d_{i+1}$. If there exists a distribution center $d_{i}$ between the locations $l_{1}$ and $l_{2}$, then the direct path between them is optimal, otherwise a detour is needed to visit a distribution center $d_{i-1}$ or $d_{i+1}$.

Theorem 1. The optimal locations $d_{1}, \ldots, d_{M}$ for $M>1$ distribution centers, when the assignment locations have uniform probability density $\rho\left(l_{1}, l_{2}\right)=1$, are equidistantly spaced at $d_{1}, d_{1}+\left(\frac{1-2 d_{1}}{M-1}\right), d_{1}+2\left(\frac{1-2 d_{1}}{M-1}\right), \ldots, d_{1}+(M-$ 1) $\left(\frac{1-2 d_{1}}{M-1}\right)=1-d_{1}$, with the boundary distance $d_{1}=$ $\frac{2-\sqrt{2}}{6-4 \sqrt{2}+2 M(\sqrt{2}-1)}$.

Proof. The locations are determined by the following optimization problem.

$$
\begin{aligned}
& \min _{d_{1}, \ldots, d_{M}} \mathbb{E}_{l_{1}, l_{2}}\left[\min _{i=1, \ldots, M}\left(\left|d_{i}-l_{1}\right|+\left|d_{i}-l_{2}\right|\right)\right] \\
& =\min _{d_{1}, \ldots, d_{M}} \int_{0}^{1} \int_{0}^{1} \min _{i=1, \ldots, M}\left(\left|d_{i}-l_{1}\right|+\left|d_{i}-l_{2}\right|\right) \mathrm{d} l_{2} \mathrm{~d} l_{1}
\end{aligned}
$$

Without loss of generality, we can assume that $d_{1} \leq$ $d_{2} \leq \cdots \leq d_{M}$. When considering using a distribution center $d_{i}$ for the assignment between $l_{1}$ and $l_{2}$, there are three possibilities, as illustrated in fig. 3 . If $d_{i}$ is between $l_{1}$ and $l_{2}$, then it is an optimal distribution center, since it is on the direct path between the two locations. Otherwise, if both locations $l_{1}, l_{2}$ belong to an interval $\left[d_{i}, d_{i+1}\right]$ for some $i$, then we need to consider both $d_{i}$ and $d_{i+1}$ as possible candidates, and the additional travel distance is $2 \cdot \min \left(\min \left(l_{1}, l_{2}\right)-d_{i}, d_{i+1}-\max \left(l_{1}, l_{2}\right)\right)$ for visiting a distribution center. Using this property, we rewrite the double integral as

$$
\begin{gathered}
\int_{0}^{1} \int_{0}^{1} \min _{i=1, \ldots, M}\left(\left|d_{i}-l_{1}\right|+\left|d_{i}-l_{2}\right|\right) \mathrm{d} l_{2} \mathrm{~d} l_{1} \\
=\int_{0}^{1} \int_{0}^{1}\left|l_{1}-l_{2}\right| \mathrm{d} l_{2} \mathrm{~d} l_{1} \\
+2 \int_{0}^{d_{1}} \int_{0}^{d_{1}}\left(d_{1}-\max \left(l_{1}, l_{2}\right)\right) \mathrm{d} l_{2} \mathrm{~d} l_{1} \\
+2 \sum_{i=1}^{M-1} \int_{d_{i}}^{d_{i+1}} \int_{d_{i}}^{d_{i+1}} \min \left(\min \left(l_{1}, l_{2}\right)-d_{i}, d_{i+1}-\max \left(l_{1}, l_{2}\right)\right) \mathrm{d} l_{2} \mathrm{~d} l_{1} \\
+2 \int_{d_{M}}^{1} \int_{d_{M}}^{1}\left(\min \left(l_{1}, l_{2}\right)-d_{M}\right) \mathrm{d} l_{2} \mathrm{~d} l_{1}
\end{gathered}
$$


Notice that the first double integral is the transportation cost for driving between $l_{1}$ and $l_{2}$, which is independent of the distribution center locations $d_{1}, \ldots, d_{M}$, thus its value will not affect the minimization problem. Let us now compute the remaining three double integrals, that represents the extra traveling cost pertaining to the distribution centers.

$$
\begin{aligned}
& \int_{0}^{d_{1}} \int_{0}^{d_{1}}\left(d_{1}-\max \left(l_{1}, l_{2}\right)\right) \mathrm{d} l_{2} \mathrm{~d} l_{1} \\
&=\int_{0}^{d_{1}}\left(\int_{0}^{l_{1}}\left(d_{1}-l_{1}\right) \mathrm{d} l_{2}+\int_{l_{1}}^{d_{1}}\left(d_{1}-l_{2}\right) \mathrm{d} l_{2}\right) \mathrm{d} l_{1} \\
&=\frac{1}{3} d_{1}^{3}
\end{aligned}
$$

Similarly,

$$
\begin{aligned}
& \int_{d_{M}}^{1} \int_{d_{M}}^{1}\left(\min \left(l_{1}, l_{2}\right)-d_{M}\right) \mathrm{d} l_{2} \mathrm{~d} l_{1} \\
&=\int_{d_{M}}^{1}\left(\int_{d_{M}}^{l_{1}}\left(l_{2}-d_{M}\right) \mathrm{d} l_{2}+\int_{l_{1}}^{1}\left(l_{1}-d_{M}\right) \mathrm{d} l_{2}\right) \mathrm{d} l_{1} \\
&=\frac{1}{3}\left(1-d_{M}\right)^{3}
\end{aligned}
$$

Finally,

$$
\begin{gathered}
\int_{d_{i}}^{d_{i+1}} \int_{d_{i}}^{d_{i+1}} \min \left(\min \left(l_{1}, l_{2}\right)-d_{i}, d_{i+1}-\max \left(l_{1}, l_{2}\right)\right) \mathrm{d} l_{2} \mathrm{~d} l_{1} \\
=\int_{d_{i}}^{\frac{d_{i}+d_{i+1}}{2}}\left(\int_{d_{i}}^{l_{1}}\left(l_{2}-d_{i}\right) \mathrm{d} l_{2}+\right. \\
\quad \int_{l_{1}}^{d_{i+1}+d_{i}-l_{1}}\left(l_{1}-d_{i}\right) \mathrm{d} l_{2}+ \\
\left.\int_{d_{i+1}+d_{i}-l_{1}}^{d_{i+1}}\left(d_{i+1}-l_{2}\right) \mathrm{d} l_{2}\right) \mathrm{d} l_{1} \\
+\int_{\frac{d_{i}+d_{i+1}}{2}\left(d _ { d _ { i + 1 } } ^ { d _ { i + 1 } } \left(d_{i}-l_{1}\right.\right.}^{d^{2}}\left(l_{2}-d_{i}\right) \mathrm{d} l_{2}+ \\
\int_{d_{i+1}+d_{i}-l_{1}}^{l_{1}}\left(d_{i+1}-l_{1}\right) \mathrm{d} l_{2}+ \\
\left.\int_{l_{1}}^{d_{i+1}}\left(d_{i+1}-l_{2}\right) \mathrm{d} l_{2}\right) \mathrm{d} l_{1} \\
6
\end{gathered}
$$

\begin{tabular}{|c|c|}
\hline$M$ & Distribution center locations $d_{1}, \ldots, d_{M}$ \\
\hline 1 & 0.5 \\
\hline 2 & $0.2929 \quad 0.7071$ \\
\hline 3 & $0.2071 \quad 0.5 \quad 0.7929$ \\
\hline 4 & $\begin{array}{llll}0.1602 & 0.3867 & 0.6133 & 0.8398\end{array}$ \\
\hline 5 & $\begin{array}{lllll}0.1306 & 0.3153 & 0.5 & 0.6847 & 0.8694\end{array}$ \\
\hline
\end{tabular}

Hence, the optimization problem for the optimal locations $d_{1}, \ldots, d_{M}$ can be written as

$$
\min _{d_{1}, \ldots, d_{M}}\left(\frac{2}{3} d_{1}^{3}+\sum_{i=1}^{M-1} \frac{1}{3}\left(d_{i+1}-d_{i}\right)^{3}+\frac{2}{3}\left(1-d_{M}\right)^{3}\right)
$$

Minimizing this expression yields the results of theorem 1 .
Remark 2. Numerically, the locations of the distribution centers for $M=1, \ldots, 5$ are

Remark 3. With uniform probability distribution, both the idling vehicle locations and the distribution center locations will be equidistantly spaced, but notice that they have different boundary conditions.

\section{B. Discrete distributions}

In the transportation system in fig. 1 there are a discrete number $C$ of cities $\mathcal{C}=\left\{c_{1}, \ldots, c_{C}\right\}$ located along the road. We now assume that both the pickup and dropoff locations are limited to the set of cities $\mathcal{C}$, thus the probability density function can be written as

$$
\rho\left(l_{1}, l_{2}\right)=\sum_{u \in \mathcal{C}} \sum_{v \in \mathcal{C}} p_{u, v} \delta\left(u-l_{1}\right) \delta\left(v-l_{2}\right)
$$

where $\delta(\cdot)$ is Dirac's delta function, $u$ and $v$ are positions of cities, and $p_{u, v}$ is the probability mass function for an assignment to be from city $u$ to city $v$. The optimal positioning of the distribution centers can be written as

$$
\begin{aligned}
& \min _{d_{1}, \ldots, d_{M}} \mathbb{E}_{l_{1}, l_{2}}\left[\min _{i=1, \ldots, M}\left(\left|d_{i}-l_{1}\right|+\left|d_{i}-l_{2}\right|\right)\right] \\
& =\min _{d_{1}, \ldots, d_{M}} \sum_{u \in \mathcal{C}} \sum_{v \in \mathcal{C}}\left[p_{u, v} \min _{i=1, \ldots, M}\left(\left|d_{i}-u\right|+\left|d_{i}-v\right|\right)\right]
\end{aligned}
$$

Proposition 3. The distribution centers can optimally be built at a subset of the cities, i.e., only locations $d_{1}, \ldots, d_{M} \in \mathcal{C}$ need to be considered.

Proof. Assume without loss of generality that $c_{1} \leq c_{2} \leq$ $\cdots \leq c_{C}$, and further assume that $\tilde{d}_{1}, \ldots, \tilde{d}_{M}$ is an optimal solution with $\tilde{d}_{i} \in\left(c_{k}, c_{k+1}\right)$ located between two cities, for some $i$ and $k$. Let $\mathcal{D} \subseteq \mathcal{C} \times \mathcal{C}$ denote the set of assignments using the distribution center $\tilde{d}_{i}$, i.e., $(u, v) \in \mathcal{D}$ if $i=$ $\arg \min _{j=1, \ldots, M}\left(\left|\tilde{d}_{j}-u\right|+\left|\tilde{d}_{j}-v\right|\right)$.

Consider now if the set of assignments $\mathcal{D}$ instead was handled by a distribution center located at $c_{k}$. Since $\tilde{d}_{i}$ is optimal, we know that

$\sum_{(u, v) \in \mathcal{D}} p_{u, v}\left(\left|\tilde{d}_{i}-u\right|+\left|\tilde{d}_{i}-v\right|\right) \leq \sum_{(u, v) \in \mathcal{D}} p_{u, v}\left(\left|c_{k}-u\right|+\left|c_{k}-v\right|\right)$.

Notice that if $u \leq \tilde{d}_{i}$ then also $u \leq c_{k}$, and if $u \geq \tilde{d}_{i}$ then $u \geq c_{k}$, since $u \in \mathcal{C}$, and similarly for $v$. Thus

$$
\sum_{\substack{(u, v) \in \mathcal{D} \\ u \leq \tilde{d}_{i} \leq v \\ \text { or } \\ v \leq \tilde{d}_{i} \leq u}} p_{u, v}\left(\left|\tilde{d}_{i}-u\right|+\left|\tilde{d}_{i}-v\right|\right)=\sum_{\substack{(u, v) \in \mathcal{D} \\ u \leq \tilde{d}_{i} \leq v \\ \text { or } \\ v \leq \tilde{d}_{i} \leq u}} p_{u, v}\left(\left|c_{k}-u\right|+\left|c_{k}-v\right|\right),
$$


so the inequality only needs to consider when $u, v \leq \tilde{d}_{i}$ or $u, v \geq \tilde{d}_{i}$. Expanding the left hand side gives

$$
\begin{aligned}
& \sum_{\substack{(u, v) \in \mathcal{D} \\
u, v \leq \tilde{d}_{i}}} p_{u, v}\left(\left|\tilde{d}_{i}-u\right|+\left|\tilde{d}_{i}-v\right|\right)+\sum_{\substack{(u, v) \in \mathcal{D} \\
u, v \geq \tilde{d}_{i}}} p_{u, v}\left(\left|\tilde{d}_{i}-u\right|+\left|\tilde{d}_{i}-v\right|\right) \\
= & \sum_{\substack{(u, v) \in \mathcal{D} \\
u, v \leq \tilde{d}_{i}}} p_{u, v}\left(\left|c_{k}-u\right|+\left|c_{k}-\tilde{d}_{i}\right|+\left|c_{k}-v\right|+\left|c_{k}-\tilde{d}_{i}\right|\right) \\
+ & \sum_{\substack{(u, v) \in \mathcal{D} \\
u, v \geq \tilde{d}_{i}}} p_{u, v}\left(\left|c_{k}-u\right|-\left|c_{k}-\tilde{d}_{i}\right|+\left|c_{k}-v\right|-\left|c_{k}-\tilde{d}_{i}\right|\right) \\
\leq & \sum_{\substack{(u, v) \in \mathcal{D} \\
u, v \leq \tilde{d}_{i}}} p_{u, v}\left(\left|c_{k}-u\right|+\left|c_{k}-v\right|\right)+\sum_{\substack{(u, v) \in \mathcal{D} \\
u, v \geq \tilde{d}_{i}}} p_{u, v}\left(\left|c_{k}-u\right|+\left|c_{k}-v\right|\right)
\end{aligned}
$$

Simplifying this inequality, we have

$$
\sum_{\substack{(u, v) \in \mathcal{D} \\ u, v \leq \tilde{d}_{i}}} p_{u, v} \leq \sum_{\substack{(u, v) \in \mathcal{D} \\ u, v \geq \tilde{d}_{i}}} p_{u, v}
$$

Repeating this argument with $c_{k+1}$ instead of $c_{k}$ yields

$$
\sum_{\substack{(u, v) \in \mathcal{D} \\ u, v \leq \tilde{d}_{i}}} p_{u, v} \geq \sum_{\substack{(u, v) \in \mathcal{D} \\ u, v \geq \tilde{d}_{i}}} p_{u, v}
$$

Together, this means that the original inequality is satisfied with equality, and hence that the location $\tilde{d}_{i}$ can be moved to either $c_{k}$ or $c_{k+1}$ without changing the value of the optimization problem. by

The locations of the distribution centers are thus given

$$
\min _{d_{1}, \ldots, d_{M} \in \mathcal{C}} \sum_{u \in \mathcal{C}} \sum_{v \in \mathcal{C}}\left[p_{u, v} \min _{i=1, \ldots, M}\left(\left|d_{i}-u\right|+\left|d_{i}-v\right|\right)\right]
$$

Remark 4. It is clear that having $M>C$ distribution centers will not improve the transportation cost, since with $M=C$, a distribution center could be built at every city.

Solving this optimization problem by brute force would consider all $\left(\begin{array}{l}C \\ M\end{array}\right)$ subsets of the cities, which grows exponentially. Instead, we propose a dynamical programming algorithm for solving this optimization problem in $O\left(C^{4}\right)$ complexity. For notational simplicity, $c \in \mathcal{C}$ can denote either the position of city $c$ or its index, as should be clear from the context. The key idea is to let cost $[m][k]$ denote the expected cost of transporting all assignments with $l_{1} \leq k$ or $l_{2} \leq k$, using at most $m$ distribution centers, where the last distribution center is located at city $k$, i.e.,

$$
\operatorname{cost}[m][k]=\min _{\substack{d_{1}, \ldots, d_{m} \in \mathcal{C} \\ d_{1} \leq \ldots \leq d_{m}=k}} \sum_{\substack{u, v \in \mathcal{C} \\ u \leq k \\ \text { or } \\ v \leq k}}\left[p_{u, v} \min _{i=1, \ldots, m}\left(\left|d_{i}-u\right|+\left|d_{i}-v\right|\right)\right]
$$

Algorithm 1 produces the optimal cost* of the solution. The optimal locations can be extracted by also memorizing which location minimizes the expression in the inner loop.

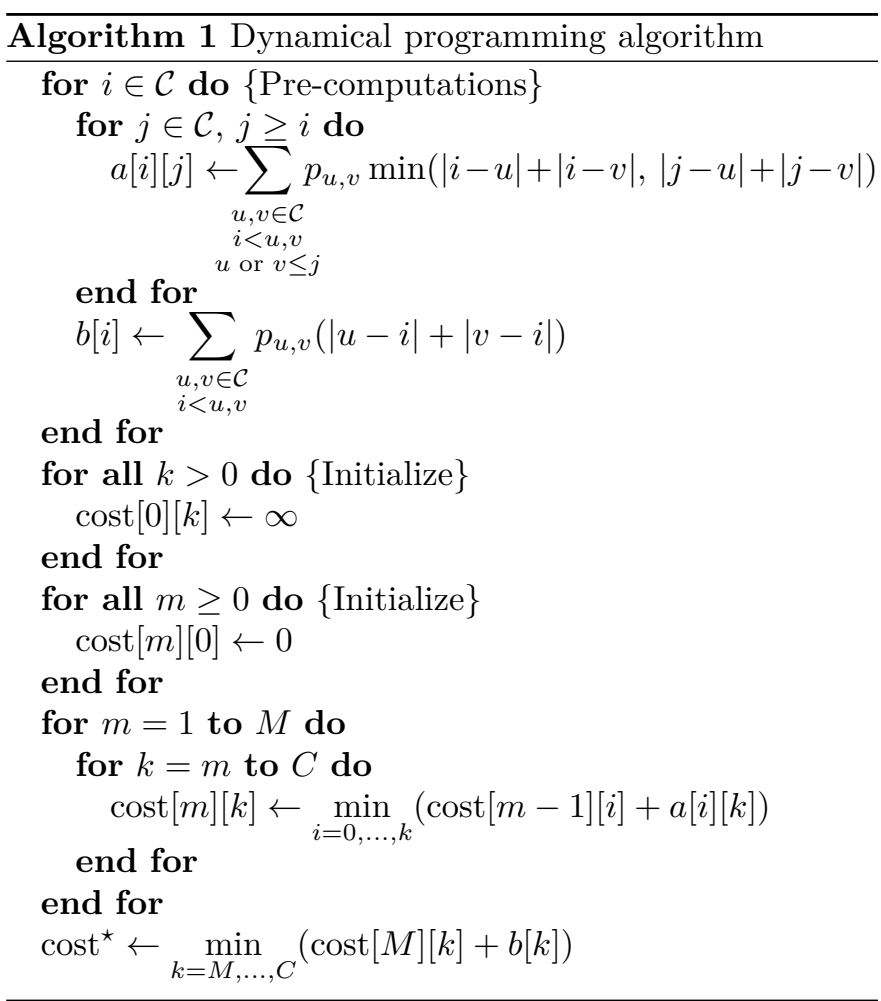

\section{Simulation Study}

In this section, we exploit the previous static optimal solutions in a dynamical transportation model with numerical simulations. Recall that the vehicles operate according the flow chart in fig. 2, and that the total time to handle an assignment consists of the time it takes to pickup the goods and the time it takes to deliver the goods to the destination, including visiting a distribution center. We thus simulate these steps independently in the following subsections.

\section{A. Idling vehicles with uniform distribution}

First, we consider transportation assignments arriving following a Poisson process with rate $\lambda$, i.e., the mean time between assignments is $1 / \lambda$, and where the pickup and drop-off locations are chosen uniformly over the interval $[0,1]$. The transportation assignments are served by $N=5$ vehicles moving with a unit speed along the road, and the objective is to minimize the average time it takes to pickup each new transport assignment. Notice that we focus on the waiting time, and ignore the fuel cost of transporting the empty vehicles in this work, in comparison to [14].

Each time a new assignment arrives, all non-occupied vehicles will be considered and the vehicle with the shortest pickup time will be selected for the assignment. The vehicle then becomes unavailable until it has completed the transport assignment. In section III we computed the optimal locations of the idling vehicles to be equidistantly spread out over the road system. We now exploit this solution as a control law for the unassigned vehicles, 


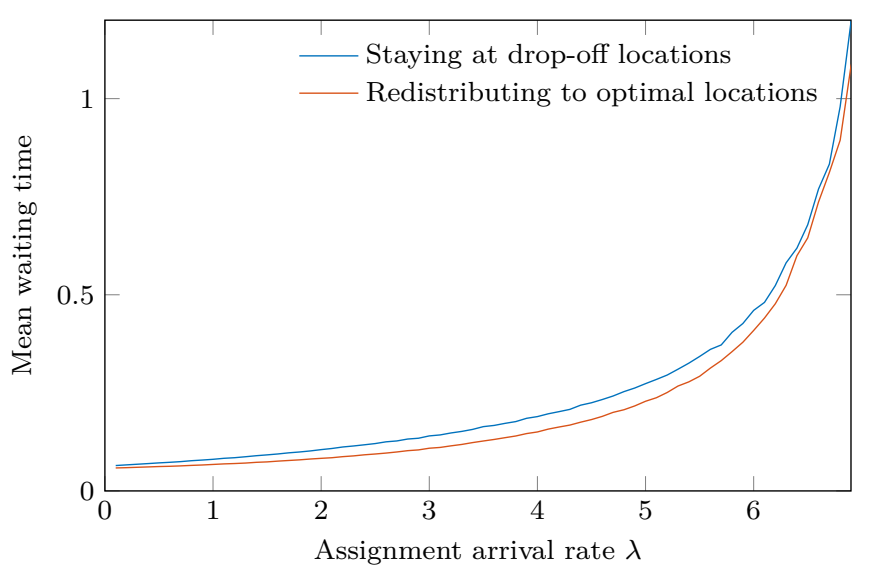

Figure 4. The average waiting time to pickup transportation assignments arriving following a Poisson process with rate $\lambda$ using 5 vehicles. Two different strategies are compared, either the vehicles stays at their drop-off location until next assignment, or they redistribute according to the optimal locations.

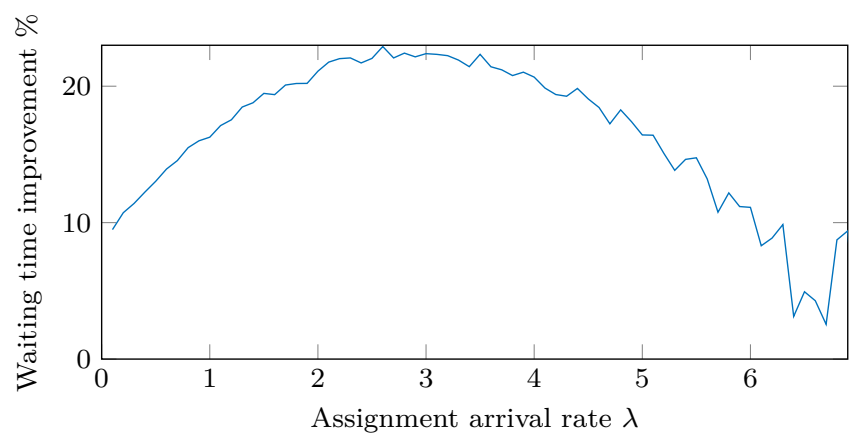

Figure 5. The improvement in the average waiting time by redistributing the vehicles towards their optimal locations, compared to staying at the drop-off location, as shown in fig. 4.

where they immediately start to redistribute themselves according to the optimal locations. For example, when one out of five vehicle is selected for a transport assignment, the remaining four available vehicles will drive towards the locations $0.125,0.375,0.625$ and 0.875 .

We compare this strategy to the base scenario, where the vehicles simply stay where they are after completing an assignment, waiting for a new assignment. The two methods are evaluated for different arrival rates $\lambda$, and for each arrival rate, the average waiting time is computed for 200000 assignments. The results are shown in figs. 4 and 5 . Notice that by exploiting the optimal vehicle location strategy, we are able to reduce the average waiting time by between $10 \%$ and $23 \%$. As seen in fig. 4 , when the arrival rate $\lambda$ approaches 7 assignments per time unit, 5 vehicles will not be sufficient to handle all assignments, which means that the waiting time starts to diverge.

\section{B. Distribution center location with discrete distribution}

Consider now the transportation stage between the pickup location and the drop-off location, which is affected

\begin{tabular}{lrrc} 
City name & Population & Distance & Relative position \\
\hline Stockholm & 923516 & $0 \mathrm{~km}$ & 0.00 \\
Södertälje & 93202 & $34 \mathrm{~km}$ & 0.07 \\
Nyköping & 54262 & $101 \mathrm{~km}$ & 0.21 \\
Norrköping & 137035 & $160 \mathrm{~km}$ & 0.34 \\
Linköping & 152966 & $198 \mathrm{~km}$ & 0.42 \\
Jönköping & 133310 & $322 \mathrm{~km}$ & 0.68 \\
Borås & 108488 & $406 \mathrm{~km}$ & 0.86 \\
Göteborg & 548190 & $470 \mathrm{~km}$ & 1.00
\end{tabular}

Major Cities along the ROAD in Fig. 1. Population Data provided by SCB [15]. Distance Given as the road Distance MEASURED FROM STOCKHOLM.

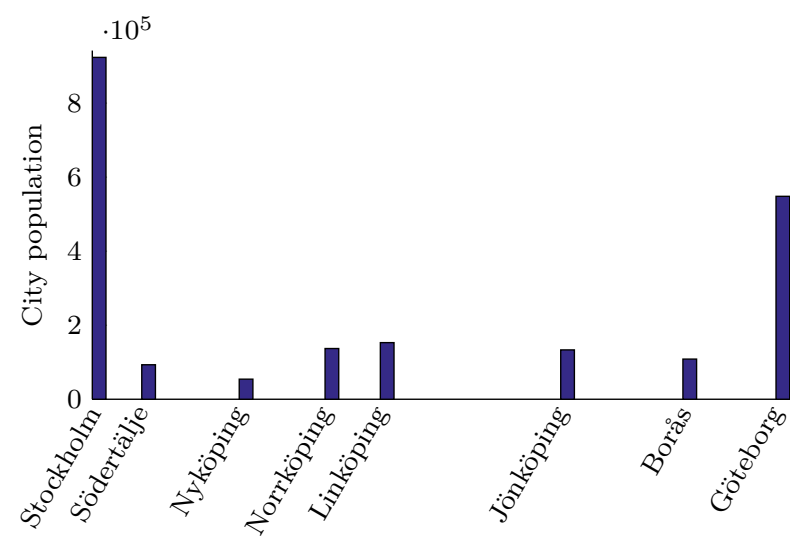

City location

Figure 6 . The city population with their relative position on the road system in fig. 1.

by the locations of distribution centers. We use the cities for the Swedish main highway, shown in fig. 1, as a discrete distribution for the assignment locations. Along this road there are 8 major cities, see table I, and the transport assignment location probabilities $p_{u, v}$ are selected proportional to the population of the cities. The population mass function is shown in fig. 6 .

The optimal distribution center locations are computed for each $M=1, \ldots, 8$ number of distribution centers, and the cities for building distribution centers is indicated in fig. 7. The range of mean traveling times is shown in fig. 8 , where the lower bound corresponds to the optimal and selected distribution centers in fig. 7. As shown, the locations of the distribution centers can significantly affect the assignment transportation time.

\section{Conclusions}

In this paper, we have considered a transportation system along a major transportation route, modeled as a one dimensional system. The goal of a real-time transport service provider is to minimize the time from the reception of a transport assignment until the delivery. This results in two separate problems, a strategy for distributing idling vehicles, and optimizing the travel time from the pickup to the drop-off locations through the construction of distribution centers. 


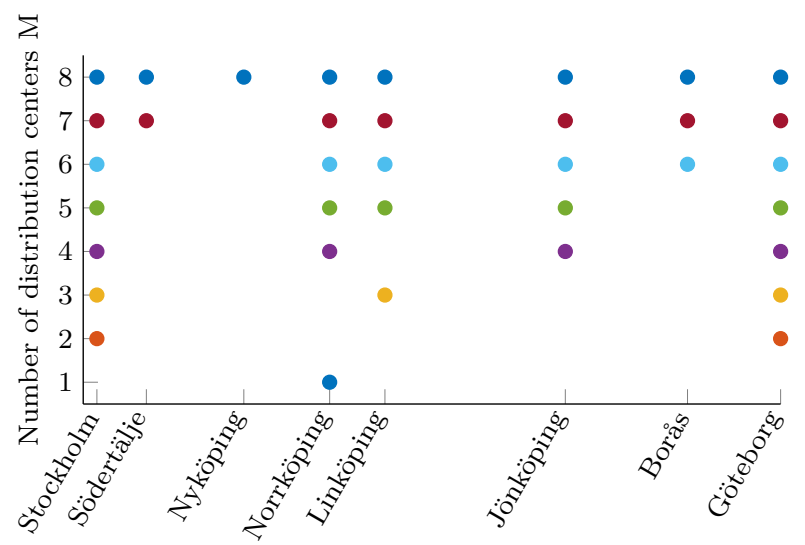

City location

Figure 7. The selected cities for building distribution centers, depending on the number of distribution centers. The markers denote where the distribution centers should be built.

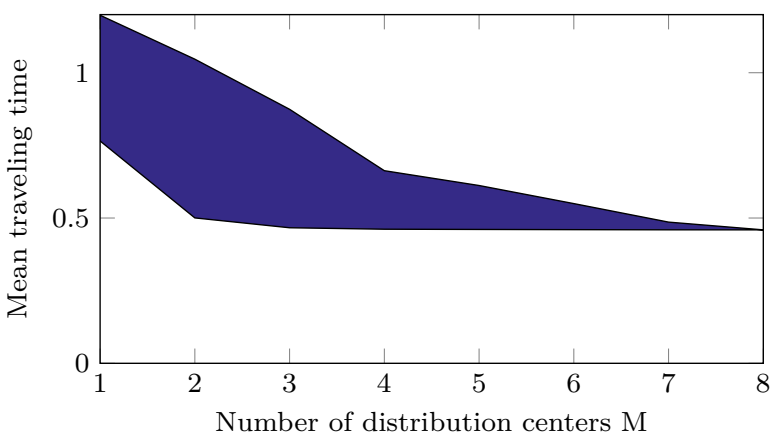

Figure 8. The range of mean traveling times for all possible choices of distribution centers. The lower bound corresponds to the optimal choice of distribution centers, shown in fig. 7 .

We formulated these problems as stochastic optimization problems, and provided explicit solutions for uniform distributions, as well as an efficient algorithm for discrete probability distributions. The methods were also evaluated with numerical simulations from a Swedish highway.

Future research directions includes allowing a vehicle to handle multiple concurrent assignments, and coordinating multiple vehicles at the distribution centers.

\section{REFERENCES}

[1] European Commission, EU transport in figures. Luxembourg: Publications Office of the European Union, 2014.

[2] P. Varaiya, "Smart cars on smart roads: problems of control," IEEE Transactions on Automatic Control, vol. 38, no. 2, pp. 195-207, 1993.

[3] R. Horowitz and P. Varaiya, "Control design of an automated highway system," Proceedings of the IEEE, vol. 88, no. 7, pp. 913-925, 2000.

[4] K.-h. Lai and T. Cheng, Just-in-time Logistics, 2009.

[5] J. Yang, P. Jaillet, and H. Mahmassani, "RealTime Multivehicle Truckload Pickup and Delivery
Problems," Transportation Science, pp. 135-148, May 2004.

[6] M. Mes, M. Van Der Heijden, and A. Van Harten, "Comparison of agent-based scheduling to look-ahead heuristics for real-time transportation problems," European Journal of Operational Research, vol. 181, no. 1, pp. 59-75, Aug. 2007.

[7] T. G. Crainic and G. Laporte, Eds., Fleet Management and Logistics. Springer, 1998.

[8] J. Perl and M. S. Daskin, "A warehouse locationrouting problem," Transportation Research Part B: Methodological, vol. 19, no. 5, pp. 381-396, Oct. 1985.

[9] C.-T. Chen, "A fuzzy approach to select the location of the distribution center," Fuzzy Sets and Systems, vol. 118, no. 1, pp. 65-73, Feb. 2001.

[10] C. Wang, W. K. Ng, and H. Chen, "From data to knowledge to action: A taxi business intelligence system," in 15th International Conference on Information Fusion, 2012, pp. 1623-1628.

[11] J. Lee, I. Shin, and G.-L. Park, "Analysis of the Passenger Pick-Up Pattern for Taxi Location Recommendation," in 4th International Conference on Networked Computing and Advanced Information Management. IEEE, Sep. 2008, pp. 199-204.

[12] T. Raviv, M. Tzur, and I. A. Forma, "Static repositioning in a bike-sharing system: models and solution approaches," EURO Journal on Transportation and Logistics, vol. 2, no. 3, pp. 187-229, Jan. 2013.

[13] K. Treleaven, M. Pavone, and E. Frazzoli, "Asymptotically Optimal Algorithms for One-to-One Pickup and Delivery Problems With Applications to Transportation Systems," IEEE Transactions on Automatic Control, vol. 58, no. 9, pp. 2261-2276, 2013.

[14] H. Terelius and K. H. Johansson, "An efficiency measure for road transportation networks with application to two case studies," in 54th IEEE Conference on Decision and Control, Dec. 2015, pp. 5149-5155.

[15] Folkmängd $i$ riket, län och kommuner, Statistiska centralbyrån (SCB), 2015. 